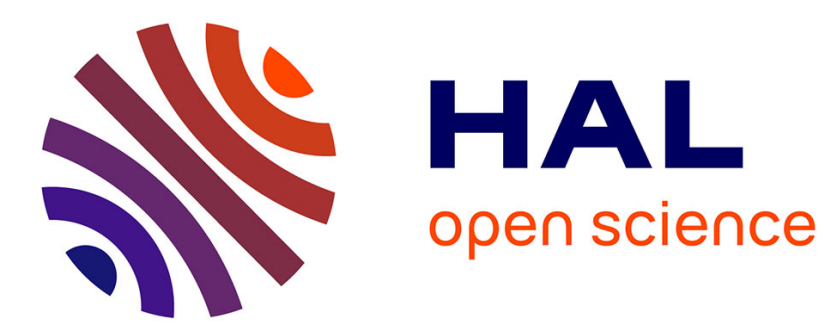

\title{
Optimized Gait Generation for Anguiliform Motion
}

Olivier Parodi, Lionel Lapierre, Bruno Jouvencel

\section{To cite this version:}

Olivier Parodi, Lionel Lapierre, Bruno Jouvencel. Optimized Gait Generation for Anguiliform Motion. OCEANS, May 2006, Singapore, Singapore. 10.1109/OCEANSAP.2006.4393885 • lirmm-00102844

\section{HAL Id: lirmm-00102844 https://hal-lirmm.ccsd.cnrs.fr/lirmm-00102844}

Submitted on 2 Oct 2006

HAL is a multi-disciplinary open access archive for the deposit and dissemination of scientific research documents, whether they are published or not. The documents may come from teaching and research institutions in France or abroad, or from public or private research centers.
L'archive ouverte pluridisciplinaire HAL, est destinée au dépôt et à la diffusion de documents scientifiques de niveau recherche, publiés ou non, émanant des établissements d'enseignement et de recherche français ou étrangers, des laboratoires publics ou privés. 


\section{Optimized Gait Generation for Anguilliform Motion}

\author{
Olivier Parodi \\ LIRMM - UMR CNRS 5506 \\ Montpellier, France \\ Email: parodi@lirmm.fr
}

\author{
Lionel Lapierre \\ LIRMM - UMR CNRS 5506 \\ Montpellier, France \\ Email: lapierre@lirmm.fr
}

\author{
Bruno Jouvencel \\ LIRMM - UMR CNRS 5506 \\ Montpellier, France \\ Email: jouvencel@lirmm.fr
}

\begin{abstract}
In this paper, we are looking for a solution to optimize the path generation of an eel-like robot. Previous works have shown that a successful approach to generate path is to control explicitly the local system curvature. This control design based on Lyapunov methods warrants the convergence of the system shape towards the desired curvature profile. Then the purpose of this work is to determine a local optimized couple of curvature parameters which are able to guarantee a fast system response, an efficient propulsion which minimizes the spent energy or both of them. This is made through three criteria which are able to give us a local solution. Finally, we present results from simulations in motion planning for a ten-link-eellike robot, to illustrate the performance of the proposed solution.
\end{abstract}

\section{INTRODUCTION}

Biologically inspired solutions are at the origin of a large new applications field. In particular in the underwater domain, it seems interesting to develop an eel-like robot. Indeed eels are able to create swirls along their body axis which ensures them a low energy consumption, efficient underwater propulsion and a high manoeuvrability [1]. For example these fishes are able to turn round with a very small radius of curvature (a fraction of their length), without losing their velocity [2].

According to the biologists, the robotics researchers could consider the body shape evolution as a propagating spatial wave. According to its position, each vertebra owns its proper signal phase. Today, two eel-like robots operate according to these results. The first one is the lamprey robot from the Northeastern University [3] and the second one is the eel-like robot from the GRASP Lab. from Harvard [4].

The first question to be solved is how to establish the model derivation of such a system. In this paper, we are considering a highly coupled, non linear hyper redundant model based on the work of K.McIsaac [5]. Many problems exist to control such a system (gait generation, control of the joint actuation, the guarantee that the system converges towards the reference path) and the purpose of this paper is to present some results of a method to find optimized values of the gait parameters. The gait generation is a very important topic. If an unappropriate gait is chosen, it will result in a poor propulsion efficiency and manoeuvrability. The solutions of the literature propose some methods which allow to control directly the joints as a trajectory tracker, or to control the joint kinematics in order to follow the desired body shape ([6], [7], [8]). W.Saintval, in [9], proposes a method to identify an optimal set of parameters according to the system situation and its main goal. Promising

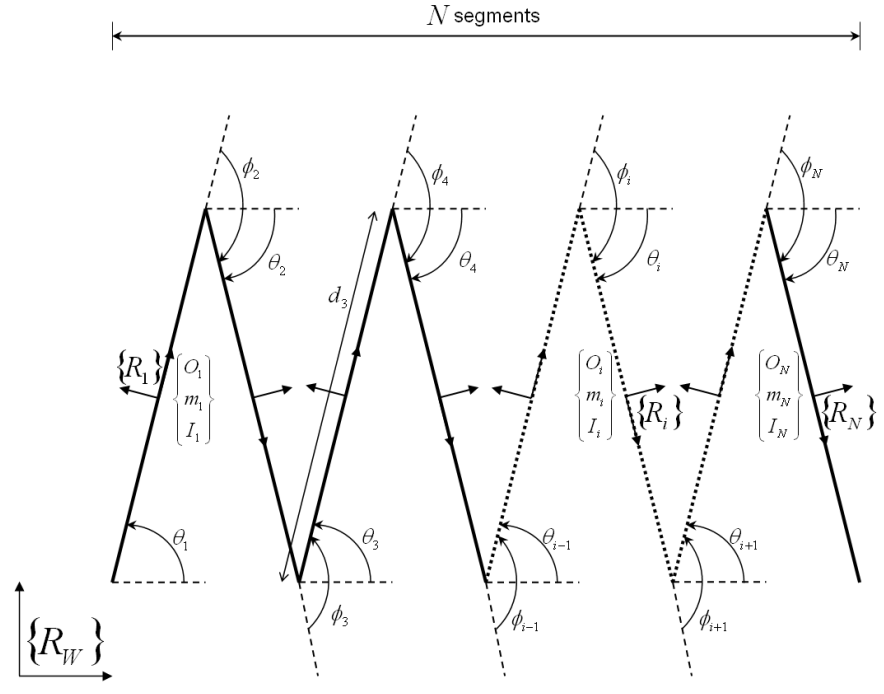

Fig. 1. Schema of the anguilliform system

results from neural approach can be found in [10] and [11].

In this paper, we propose a method to find gait optimized local parameters based upon three criteria. These criteria allow us to choose an optimal couple of parameters which could be either a system time reaction optimization, a low energy consumption optimization or the two optimizations combined. The system is driven according to a reference body curvature which is not time dependent and that explicitly takes the system dynamics into account as described in [13]. The system convergence is warranted by using Lyapunov methods in the derivation model.

This paper is organized as follows: chapter II describes the system modeling, chapter III introduces the control design, chapter IV is about the optimization method according to some criteria and finally chapter $\mathrm{V}$ indicates some simulation results to illustrate the performances of our method.

\section{SYSTEM MODELING}

\section{A. Representation}

We are modeling an anguilliform robot as a sequel of $N$ planar articulated rigid segments. As we can see on figure 1 the robot structure is considered as:

- we also call $\left\{R_{W}\right\}$ the inertial frame, $\left\{R_{1}\right\}$ the frame attached to the head and $\left\{R_{i, i=2 \ldots N}\right\}$ the body frames attached to each segment. 
- a chain of $N$ links of length $d_{i, i=1 \ldots N}$

- each link $i$ has a a proper mass called $m_{i, i=1 \ldots N}$

- each link $i$ has a moment of inertia called $I_{i, i=1 \ldots N}$ expressed in the center $O_{i, i=1 \ldots N}$

- $\theta_{i, i=1 \ldots N}$ is the relative angle between the link $i$ and the $O_{x}$ axe of the inertial frame

- $\phi_{i, i=2 \ldots N}$ is the relative angle between the link $i-1$ and $i$

Now, we are able to establish the dynamic model expressing the articular efforts according to the hydrodynamic parameters of the system and its intrinsic properties.

\section{B. Geometric Model}

Considering the coordinates of the center of the segments expressed in the inertial frame according to the head coordinates and the relative angle between the head and the $O_{x}$ axe of inertial frame, we obtain:

$$
\left\{\begin{array}{l}
x_{i}=x_{1}+\frac{d_{1}}{2} \cos \theta_{1}+\sum_{j=2}^{i-1}\left(d_{j} \cos \theta_{i}\right)+\frac{d_{i}}{2} \cos \theta_{i} \\
y_{i}=y_{1}+\frac{d_{1}}{2} \sin \theta_{1}+\sum_{j=2}^{i-1}\left(d_{j} \sin \theta_{i}\right)+\frac{d_{i}}{2} \sin \theta_{i} \\
\theta_{i}=\theta_{1}+\sum_{k=2}^{i}\left(\phi_{k}\right)
\end{array}\right.
$$

Knowing:

- the position $\left(x_{1}, y_{1}\right)^{T}$ of the head's center in the inertia frame,

- the relative angle $\theta_{1}$ between the head and the horizontal axe of the inertia base $\left\{R_{W}\right\}\left(O_{x}\right)$,

- all the relative angles $\phi_{k, k=2 \ldots N}$ between the segments, we obtain:

- the position $\left(x_{i, i=2 \ldots N}, y_{i, i=2 \ldots N}\right)^{T}$ of all the segments' center in the inertial frame $\left\{R_{W}\right\}$, according to equation 1.

\section{Kinematic Model}

The derivation of the system (1) yiealds the expression of the velocities in the inertial frame $\left(\dot{x}_{i=1 \ldots N}, \dot{y}_{i=1 \ldots N}, \dot{\theta}_{i=1 \ldots N}\right)$. In order to obtain these velocities in the body frame, we establish the rotation matrices $T_{W}^{i}$ between $\left\{R_{W}\right\}$ and $\left\{R_{i}\right\}$. We also define two groups of kinematic variables called configuration variables $\dot{q}$ and shape variables $\dot{s}$ as follows:

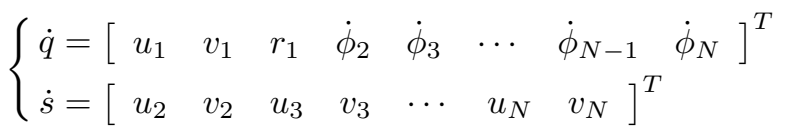

where $u_{i}, v_{i}$ and $r_{i}$ are respectively the forward velocity, the side-slip velocity and the rotational velocity of each link $i$ expressed in the body frames $\left\{R_{i}\right\}_{i=1 \ldots N}$. We remark that group $q$ defines a necessary and sufficient set to describe the system situation. So we deduce the expression of the kinematic model:

$$
\left\{\begin{aligned}
u_{i} & =u_{1} \cos \left(\theta_{i}-\theta_{1}\right)+v_{1} \sin \left(\theta_{i}-\theta_{1}\right) \\
& +\frac{r_{1} d_{1}}{2} \sin \left(\theta_{i}-\theta_{1}\right)+\sum_{j=2}^{i-1}\left(d_{j} r_{j} \sin \left(\theta_{i}-\theta_{j}\right)\right) \\
v_{i} & =-u_{1} \sin \left(\theta_{i}-\theta_{1}\right)+v_{1} \cos \left(\theta_{i}-\theta_{1}\right) \\
& +\frac{r_{1} d_{1}}{2} \cos \left(\theta_{i}-\theta_{1}\right)+\sum_{j=2}^{i-1}\left(d_{j} r_{j} \cos \left(\theta_{i}-\theta_{j}\right)\right) \\
& +\frac{r_{i} d_{i}}{2} \\
r_{i} & =r_{1}+\sum_{j=2}^{i} \dot{\phi}_{j}
\end{aligned}\right.
$$

\section{Dynamic Model Elaboration}

1) Hydrodynamic Approximations: we model each link of the eel-like robot as a cylinder of length $d_{i}$, radius $L_{i}$, mass $m_{i}$ and moment of inertia $I_{i}$. We consider 3 types of significant hydrodynamic forces applied on the system:

- perpendicular drag effect: we assume that differential pressure only acts perpendicularly to the body. These forces are those which produce the system thrust. We assume the approximations used in [7]:

$$
F_{i}^{v}=-\mu_{i} v_{i}
$$

where $F_{i}^{v}$ is the perpendicular drag force acting in the link $i$, moving with a slide-slip velocity $v_{i} ; \mu_{i}$ is the perpendicular drag force coefficient of the considered system element.

- parallel drag effect: assuming the streamlined nature of the eel's body, the friction coefficient in the body direction is negligible with respect to the perpendicular direction ([7], [11]). Nevertheless, this effect is significant on the first link (also called head) for forward movement, and on the last segment (tail) for backward movement.

$$
\begin{aligned}
& F_{1}^{u}= \begin{cases}-\nu_{1} u_{1} & \text { if } u_{1}>0 \\
0 & \text { if } u_{1} \leq 0\end{cases} \\
& F_{N}^{u}= \begin{cases}-\nu_{N} u_{N} & \text { if } u_{1}<0 \\
0 & \text { if } u_{1} \geq 0\end{cases} \\
& F_{i=2, \cdots, N-1}^{u}=0
\end{aligned}
$$

where $F_{1}^{u}$ and $F_{N}^{u}$ produce the parallel drag effect acting on the head and the tail respectively, $\nu_{i, i=1 . . N}$ are the parallel drag coefficients, and $u_{i}$ denotes the forward speed of the $i^{\text {th }}$ element.

- added mass: the added mass term is estimated considering the cylindrical shape of the system components. We refer to [12] for an approximation of this parameter effect. The inertial matrix for the $i^{t h}$ element, expressed in the body frame $\left\{R_{i}\right\}$ is denoted:

$$
M_{i}=\left[\begin{array}{ccc}
m_{i}^{x} & 0 & 0 \\
0 & m_{i}^{y} & 0 \\
0 & 0 & I_{i}
\end{array}\right]
$$


where $m_{i}^{x}$ and $m_{i}^{y}$ are the wet masses (inertial and added mass) in the $x$ and $y$ direction of the body frame $\left\{R_{i}\right\}$, and $I^{i}$ is the inertial wet moment of the $i^{\text {th }}$ element.

2) Lagrangian Elaboration: According to the results described in [13] (II.B), the Lagrangian of the system is:

$$
L=\frac{1}{2} \dot{q}^{T}\left(I+\beta^{T} M \beta\right) \dot{q}
$$

with:

$$
\text { • I=[ }\left[\begin{array}{ccccccc}
m_{1}^{x} & 0 & \cdots & \cdots & \cdots & \cdots & 0 \\
0 & m_{1}^{y} & 0 & \cdots & \cdots & \cdots & 0 \\
\vdots & 0 & a_{1} & a_{2} & a_{3} & \cdots & a_{N} \\
\vdots & \vdots & a_{2} & a_{2} & a_{3} & & \vdots \\
\vdots & \vdots & a_{3} & a_{3} & a_{3} & & \vdots \\
\vdots & \vdots & \vdots & & & \ddots & \vdots \\
0 & 0 & a_{N} & \cdots & \cdots & \cdots & a_{N}
\end{array}\right]
$$

where: $a_{i}=\sum_{k=i}^{N} I_{k}$

$$
\text { - } M=\left[\begin{array}{ccccccc}
m_{2}^{x} & 0 & \ldots & \ldots & \ldots & \ldots & 0 \\
0 & m_{2}^{y} & \ddots & & & & \vdots \\
\vdots & \ddots & m_{3}^{x} & \ddots & & & \vdots \\
\vdots & & \ddots & m_{3}^{y} & \ddots & & \vdots \\
\vdots & & & \ddots & \ddots & \ddots & \vdots \\
\vdots & & & & \ddots & m_{N}^{x} & 0 \\
0 & \ldots & \ldots & \ldots & \ldots & 0 & m_{N}^{y}
\end{array}\right]
$$

- $\beta=\beta_{\theta} T_{\theta}^{\phi}$ where:

$$
\begin{aligned}
& \beta_{\theta}=\left[\begin{array}{ccccccc}
c_{1}^{2} & s_{1}^{2} & \frac{d_{1}}{2} s_{1}^{2} & 0 & \cdots & \cdots & 0 \\
-s_{1}^{2} & c_{1}^{2} & \frac{d_{1}}{2} c_{1}^{2} & \frac{d_{2}}{2} & 0 & & \vdots \\
c_{1}^{3} & s_{1}^{3} & \frac{d_{1}}{2} s_{1}^{3} & d_{2} s_{2}^{3} & 0 & \ddots & \vdots \\
-s_{1}^{3} & c_{1}^{3} & \frac{d_{1}}{2} c_{1}^{3} & d_{2} c_{2}^{3} & \frac{d_{3}}{2} & 0 & \vdots \\
\vdots & \vdots & \vdots & \vdots & \vdots & \vdots & \vdots \\
c_{1}^{N} & s_{1}^{N} & \frac{d_{1}}{2} s_{1}^{N} & d_{2} s_{2}^{N} & d_{3} s_{3}^{N} & 0 & 0 \\
-s_{1}^{N} & c_{1}^{N} & \frac{d_{1}}{2} c_{1}^{N} & d_{2} c_{2}^{N} & d_{3} c_{3}^{N} & \ldots & \frac{d_{N}}{2}
\end{array}\right] \\
& T_{\theta}^{\phi}=\left[\begin{array}{cccccc}
1 & 0 & \cdots & \cdots & \cdots & 0 \\
0 & 1 & \ddots & & & \vdots \\
\vdots & 0 & 1 & \ddots & & \vdots \\
\vdots & \vdots & \vdots & \ddots & \ddots & \vdots \\
\vdots & \vdots & \vdots & & \ddots & 0 \\
0 & 0 & 1 & \cdots & \cdots & 1
\end{array}\right] \\
& \text { with } c_{k}^{i}=\cos \left(\theta_{i}-\theta_{k}\right) \text { and } s_{k}^{i}=\sin \left(\theta_{i}-\theta_{k}\right)
\end{aligned}
$$

3) Dynamical Model: The dynamical model is derived by using:

$$
F^{e x t}=\frac{d}{d t} \frac{\partial L}{\partial \dot{q}}-\frac{\partial L}{\partial q}
$$

to obtain:

$$
F^{e x t}=\left(\beta^{T} M \dot{\beta}\right) \dot{q}+\left(I+\beta^{T} M \beta\right) \ddot{q}
$$

And the expression $F^{e x t}=F_{1}+\beta^{T} F_{2}$ yields

$$
\begin{aligned}
\ddot{q} & =\left(I+\beta^{T} M \beta\right)^{-1} F_{1} \\
& +\left(I+\beta^{T} M \beta\right)^{-1} \beta^{T} F_{2} \\
& -\left(I+\beta^{T} M \beta\right)^{-1}\left(\beta^{T} M \dot{\beta}\right) \dot{q}
\end{aligned}
$$

where $F_{1}=\left[F_{1}^{u}, F_{1}^{v}, 0, \tau_{2}, \cdots, \tau_{N}\right]^{T}$ are the forces that act in the dimensions defined by the configuration variable $q$, and $F_{2}=\left[F_{2}^{u}, F_{2}^{v}, \cdots, F_{N}^{u}, F_{N}^{v}\right]^{T}$ contains forces that act in the dimension defined by the dependent variables $s$. We also define $\Gamma=\left[\tau_{1}, \cdots, \tau_{N}\right]^{T}$ which is the torques control vector.

\section{Control Design}

The system thrust is ensured by a periodic signal propagation along the body: this signal is called gait. Two approaches at least are possible to control such a system:

- we can impose explicitly a sinusoidal reference of the form: $\phi_{i}(t)=A_{i} \sin \left(\omega_{i} t+\psi_{i}\right)$ to the joint actuators, each link having its own phase according to its situation as in [5]. The problem consists in adjusting the gait parameters $A_{i}, \omega_{i}$ and $\psi_{i}$.

- We can impose a spatial curvature to the system and we can make the shape of the robot converge towards this curvature. Then we intend to make the system move along this curvature profile.

We propose to use this second approach for the gait generation which is not time dependent and which allows us to control the local curvature of the system explicitly. This leads to an autonomous system on which we can use reserved mathematic tools. With the following notations:

$$
\left\{\begin{array}{l}
\Xi=\left(I+\beta^{T} M \beta\right)^{-1} \\
B=\beta^{T} F_{2}-\left(\beta^{T} M \dot{\beta}\right) \dot{q}
\end{array}\right.
$$

Equation (3) leads to: $\ddot{q}=\Xi\left(F_{1}+B\right)$. Decomposing $\Xi F_{1}$ and $B$ matrixes as follows:

$$
\begin{gathered}
\Xi=\left[\begin{array}{cc}
\Xi_{1} & \Xi_{2} \\
(3 \times 3) & (3 \times(N-1)) \\
\Xi_{3} & \Xi_{4} \\
((N-1) \times 3) & ((N-1) \times(N-1))
\end{array}\right] B=\left[\begin{array}{c}
B_{1} \\
(3 \times 1) \\
B_{2} \\
((N-1) \times 1)
\end{array}\right] \\
F_{1}=[\underbrace{f_{1}}_{3 \times 1}, \underbrace{\tau_{1}, \cdots, \tau_{N}}_{\Gamma}]^{T}
\end{gathered}
$$

leads to a first control equation:

$$
\Gamma=\Xi_{4}^{-1}\left(\ddot{\phi}-\Xi_{3}\left(f_{1}+B_{1}\right)\right)-B_{2}
$$

\section{A. Control Equation}

Let $s_{i}$ be the curvilinear coordinate of the $i^{t h}$ articulation. $s_{1}$ denotes the curvilinear position of the head and $s_{N+1}$ is the one of the tail. Then at $t=0$, the initial values are $s_{1(t=0)}=$ 
$0, s_{2(t=0)}=d_{1}, s_{3(t=0)}=d_{1}+d_{2}, \cdots, s_{N(t=0)}=\sum_{k=1}^{N+1} d_{k}$. In the following sections, the upper scripts $(.)^{R}$ and $(.)^{E}$ indicate respectively the reference and the eel. Let $C_{i}$ be the curvature for the $i^{t h}$ articulation, estimated with:

$$
C_{i}^{E}=\frac{\theta_{i}-\theta_{i-1}}{d_{i}+d_{i+1}}=\frac{\phi_{i}}{d_{i}+d_{i+1}}
$$

The control objective is thus to drive the system curvature $C_{i}^{E}$ towards the reference $C_{i}^{R}$ for each articulation $i$.

1) Kinematic Reference Derivation: Let $V_{1}=$ $\frac{1}{2} \sum_{i=2}^{N}\left(C_{i}^{R}-C_{i}^{E}\right)^{2} \quad$ be a Lyapunov candidate. Straightforward computation shows that the choice

$\dot{C}_{i}^{E}=\dot{C}_{i}^{R}+K_{1}\left(C_{i}^{R}-C_{i}^{E}\right)^{2}{ }_{i=2, \ldots, N} K_{1}$ positive definite yields $\dot{V}_{1}=-K_{1} \sum_{i=2}^{N}\left(C_{i}^{R}-C_{i}^{E}\right)^{2} \leq 0$. Let $\dot{C}_{i}^{K I N}$ be this particular $\dot{C}_{i}^{E}$ called the kinematic reference for the dynamic control:

$$
\dot{C}_{i}^{K I N}=\dot{C}_{i}^{R}+K_{1}\left(C_{i}^{R}-C_{i}^{E}\right){ }_{i=2, \ldots, N}
$$

2) Dynamic Control Design: Let $V_{2}=$ $\frac{1}{2} \sum_{i=2}^{N}\left(\dot{C}_{i}^{K I N}-\dot{C}_{i}^{E}\right)^{2}$ be a Lyapunov candidate. The derivation of $V_{2}$ shows that the choice

$$
\ddot{C}_{i}^{E}=\ddot{C}_{i}^{K I N}+K_{2}\left(\dot{C}_{i}^{K I N}-\dot{C}_{i}^{E}\right) K_{2} \text { positive defined }
$$

yields $V_{2} \leq 0$. Let $\ddot{C}_{i}^{D Y N}$ be this particular $\ddot{C}_{i}^{E}$ called the dynamic reference

$$
\ddot{C}_{i}^{D Y N}=\ddot{C}_{i}^{K I N}+K_{2}\left(\dot{C}_{i}^{K I N}-\dot{C}_{i}^{E}\right)
$$

This leads to a desired joint acceleration

$$
\ddot{\phi}_{i}^{R}=\left(d_{i}+d_{i-1}\right) \ddot{C}_{i}^{D Y N}
$$

Yielding the final control expression:

$$
\begin{aligned}
\Gamma= & \Xi_{4}^{-1}\left[\left(\ddot{C}_{i}^{R}+\left(K_{1}+K_{2}\right)\left(\dot{C}_{i}^{R}-\dot{C}_{i}^{E}\right)\right.\right. \\
& \left.+K_{1} K_{2}\left(C_{i}^{R}-C_{i}^{E}\right)\right) \times\left(d_{i}+d_{i+1}\right) \\
& \left.-\Xi_{3}\left(f_{1}+B_{1}\right)\right]-B_{2}
\end{aligned}
$$

This control imposes the system to slide on the curvature reference with a curvilinear velocity $\dot{s}$. Now we must choose a curvature which will impose periodic movements to the eellike robot. We thus introduce such a reference as follows:

$$
C_{i}^{R}=A \sin \left(\omega_{i}\right)
$$

$\Gamma$ is now entirely defined. The challenge now consists in finding an appropriate set of parameters $(A, \omega)$ which do not generate structural conflicts and which will induces some good performances to the system.

\section{B. Command Law Constraints}

1) Mechanical Constraints: One of the first questions to be posed is to know which parameters $(A, \omega)$ to choose. Indeed some configurations resulting from a bad choice of parameters are not acceptable for the robot. These shape configurations
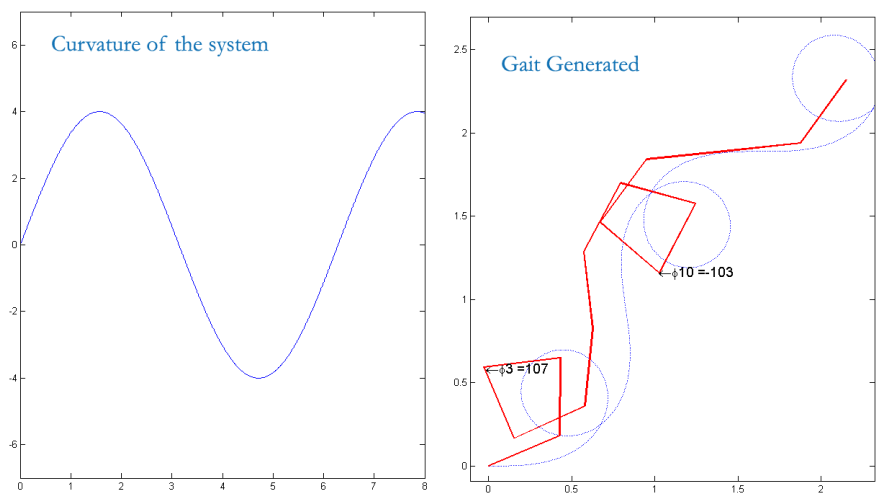

Fig. 2. Mechanical Problem

are in fact some structural constraints dependent on the robot intrinsic properties. There are 2 at least:

- the first constraint is to prevent the robot from making loops, i.e we must eliminate the sets of parameters which generate crossings of links

- the second one comes from the mechanical structure. Indeed it's necessary to ensure that the value of two consecutive angles is not higher than what the robot could handle.

On figure 2, we can see the curvature ( $A=4$ and $\omega=1$ ) of the system on the left side, the gait generated from this curvature and the shape configuration of the eel robot on the other size; we have imposed a $\phi_{\max }=102.5^{\circ}$ to the system. The robot is submitted to both the mechanical constraints. Through this example we realize well that some shapes configuration and consequently some sets of parameters $(A, \omega)$ are proscribed.

\section{2) Shape Convergence Constraint:}

a) Problem Position: Another problem concerns the robot shape convergence. Indeed according to the chosen set of parameters, the robot is more or less able to converge towards the gait reference generated from the curvature. The purpose is thus to eliminate the set of $(A, \omega)$ which generate curvature aberrations. On figure 3 we can see that it's physically impossible for the robot to respect the desired curvature. This leads us to elaborate a convergence criterion.

b) Convergence Criterion: In order to estimate the variation between the desired curvature and the position of the robot, we are going to estimate the area between the desired shape configuration and the real one. We use a numerical method to evaluate the area $S$ : we calculate the absolute value of the difference between the desired shape integrals and the eel's one (figure 2 . In the equation 6 The $^{E}$ and the ${ }^{C}$ notations 


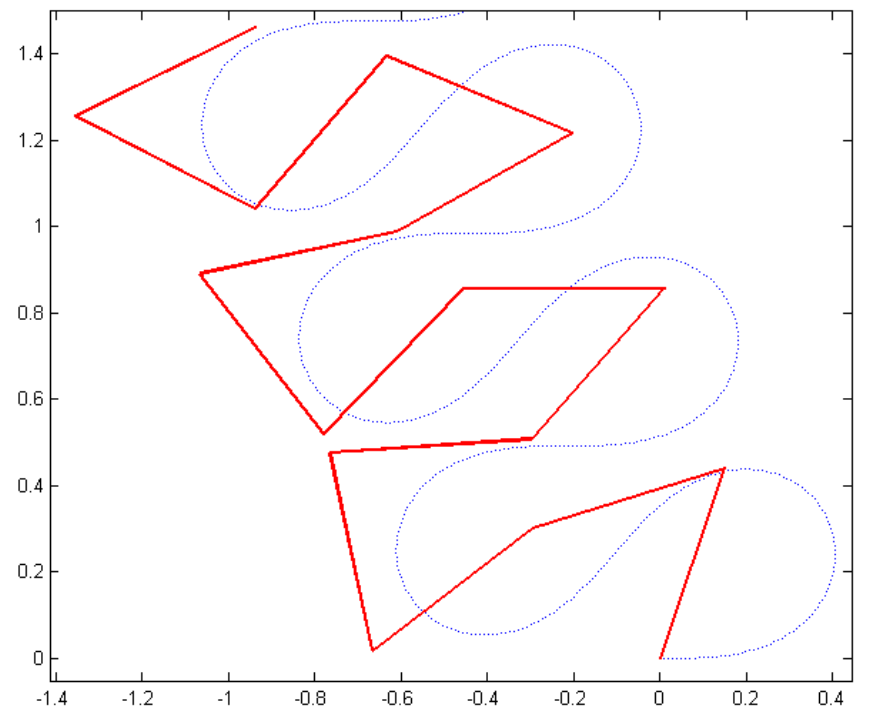

Fig. 3. Problem of the Shape convergence

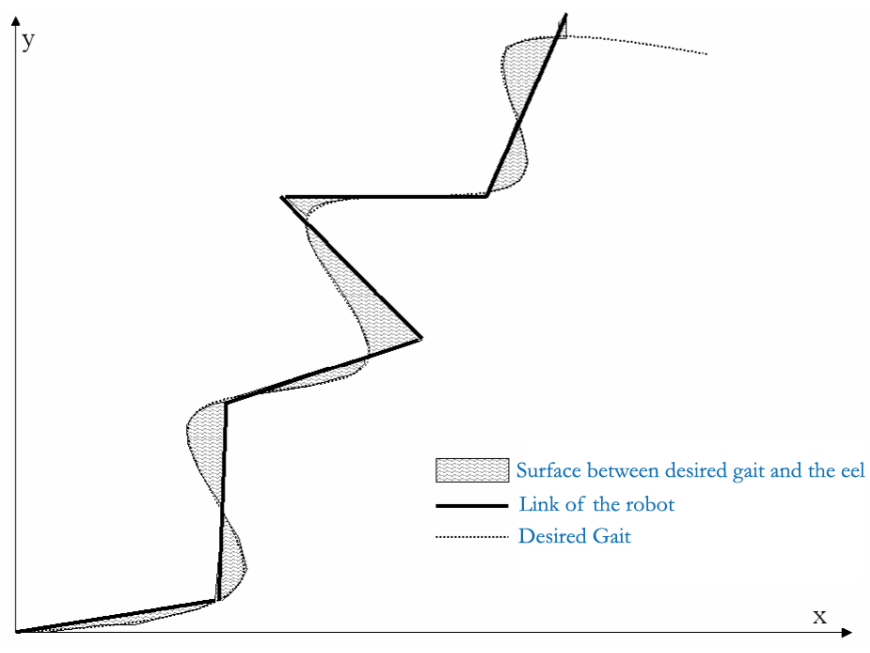

Fig. 4. Convergence Criterion

are respectively used for the eel and for the desired curvature.

$$
\begin{aligned}
S_{k+1} & =\left(S_{k}^{E}+\left\|x_{k+1}^{E}-x_{k}^{E}\right\|\left(\min \left(\left\|y_{k}^{E}\right\|,\left\|y_{k}^{E}\right\|\right)\right.\right. \\
& \left.\left.+\frac{\max \left(\left\|y_{k}^{E}\right\|,\left\|y_{k}^{E}\right\|\right)-\min \left(\left\|y_{k}^{E}\right\|,\left\|y_{k}^{E}\right\|\right)}{2}\right)\right) \\
& -\left(S_{k}^{C}+\left\|x_{k+1}^{C}-x_{k}^{C}\right\|\left(\min \left(\left\|y_{k}^{C}\right\|,\left\|y_{k}^{C}\right\|\right)\right.\right. \\
& \left.\left.+\frac{\max \left(\left\|y_{k}^{C}\right\|,\left\|y_{k}^{C}\right\|\right)-\min \left(\left\|y_{k}^{C}\right\|,\left\|y_{k}^{C}\right\|\right)}{2}\right)\right)
\end{aligned}
$$

c) Results and Thresholding: Thanks to this criterion, we obtain figure 5 which shows that, according to the choice of the sets of parameters, the structure converges more or less towards the desired shape. The next step consists in

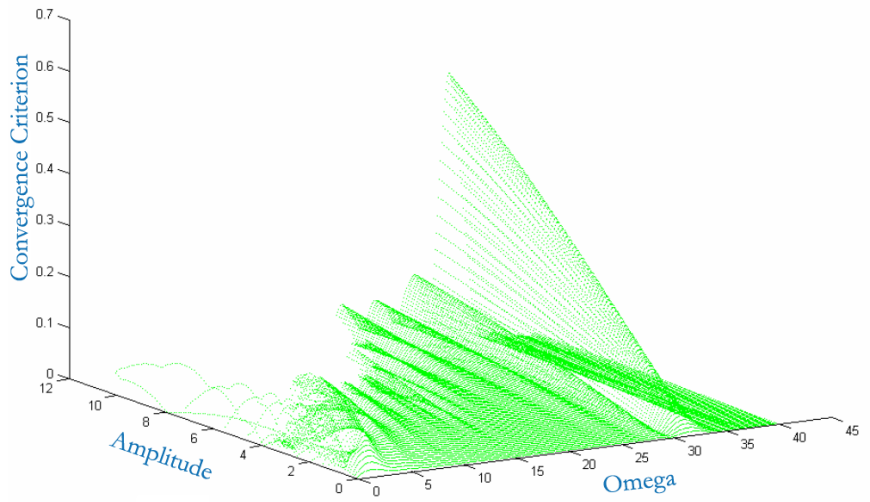

Fig. 5. Convergence Criterion according to $A$ and $\omega$
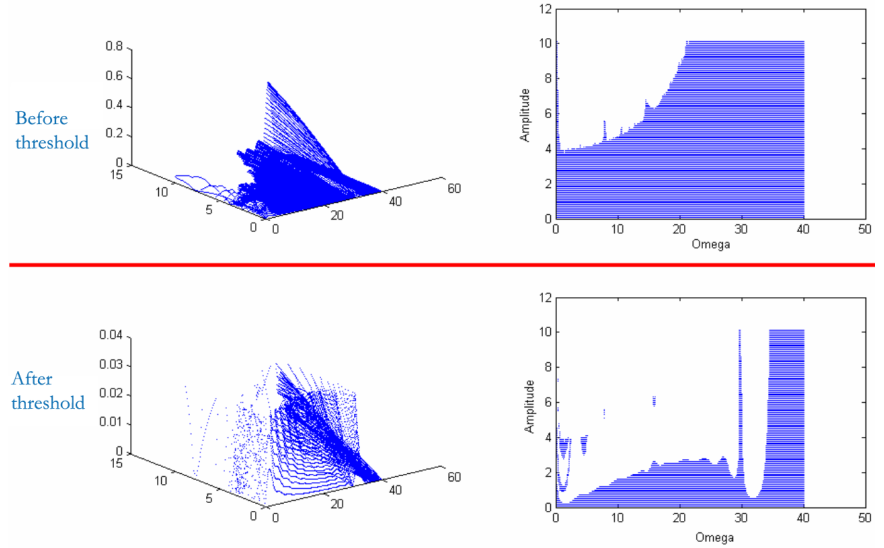

Fig. 6. Threshold

thresholding the convergence criterion in order to eliminate the undesirable parameters. So, for example, we keep $40 \%$ of the couples for which the convergence criterion is closest to 0 as shown on figure 6 . This allows us to reduce the research domain for the appropriate sets of parameters. We note that the domain of the selected couples is not connected.

\section{Elaborating CRITERiA}

We have shown previously that some sets $(A, \omega)$ have to be proscribed. Now we must determine the couples to be used in our Matlab simulator among those which are pre-selected. In order to do this, 3 criteria will be considered:

- energy criterion

- system's reactivity criterion

- criterion combining both of them.

So according to the considered applications, we should be able to choose the optimal set.

\section{A. Energy Criterion}

The purpose is to elaborate a criterion analogous with the system energy:

$$
J_{1}=\sum_{t=0}^{f t} \sum_{i=1}^{N}\left\|\Gamma_{i}\right\|^{2}
$$




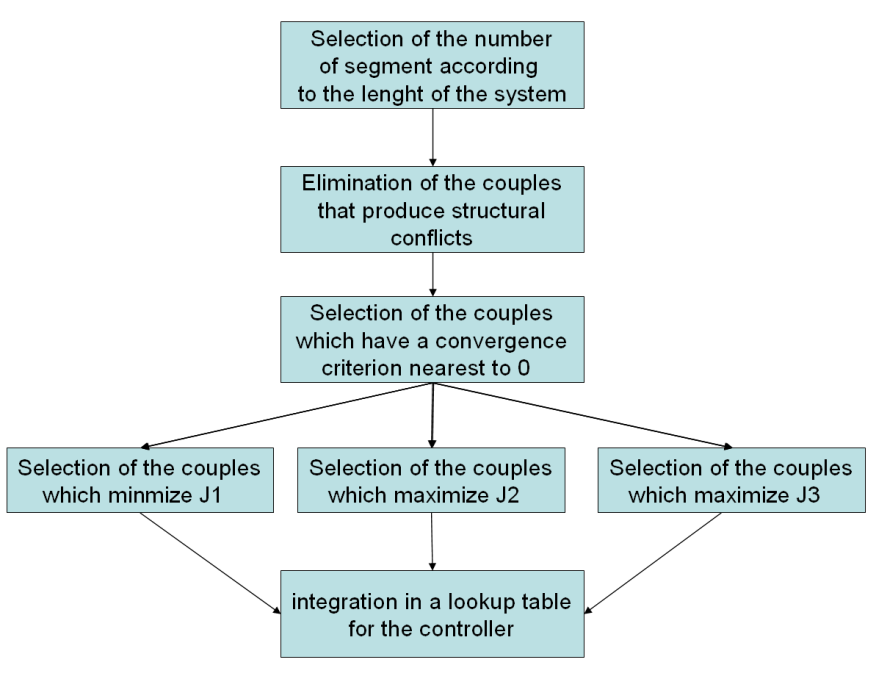

Fig. 7. Stages of the method

where $f t$ is the final time.

We are searching the sets of parameters which minimize this criterion.

\section{B. Criterion of the System's Reactivity}

We intend to elaborate a criterion that capture the ability of the system to reach as fastest as possible its maximum velocity. So we consider:

$$
J_{2}=\sum_{t=0}^{f t} \sum_{i=1}^{N}\left\|u_{i}\right\|^{2}
$$

We are searching the sets of parameters which maximize this criterion.

\section{Combined Criterion}

If the purpose is now to find the best compromise between $J_{1}$ and $J_{2}$, we have to maximize the criterion $J_{3}$ defined as follows:

$$
J_{3}=\frac{J_{2}}{J_{1}}
$$

Using these 3 criteria, we have got the necessary tools to determine the sets $(A, \omega)$ to be given to the controller according to the desired velocity and the desired type of optimization that is wished.

\section{Method of Election And Results}

In order to illustrate our purpose, we are summing up the different stages of the method in figure 7.

\section{A. Election of the couples}

The first step consists in selecting an appropriate number of links according to the robot's length. In order to do this, we trace the evolution of the convergence criterion according to the segments number, as it is shown on figure 8 (length of the eel-robot: $2 \mathrm{~m}, \phi_{\max }=45^{\circ}$ ). We observe on figure 8 that by choosing a 0.2-length link, the system has a good shape

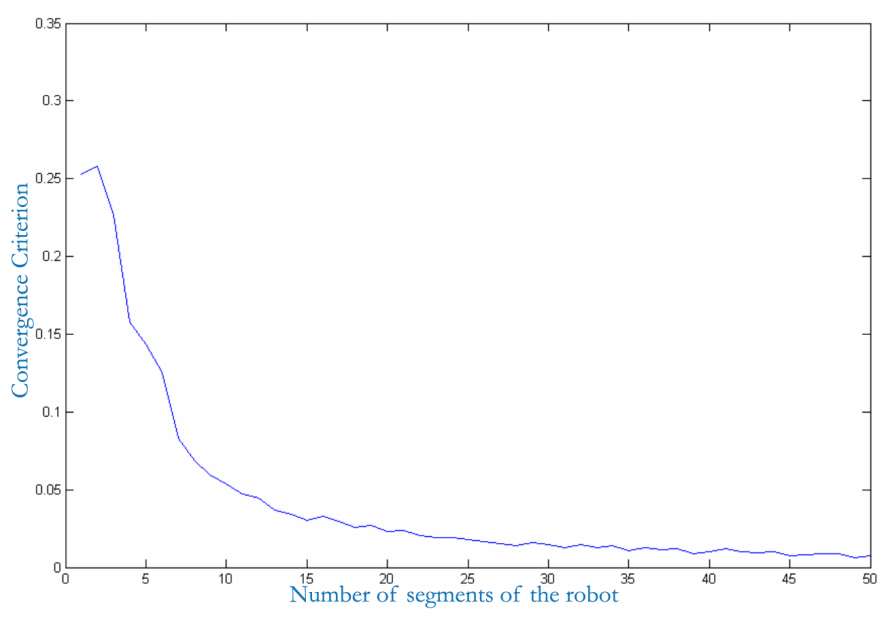

Fig. 8. Convergence criterion evolution
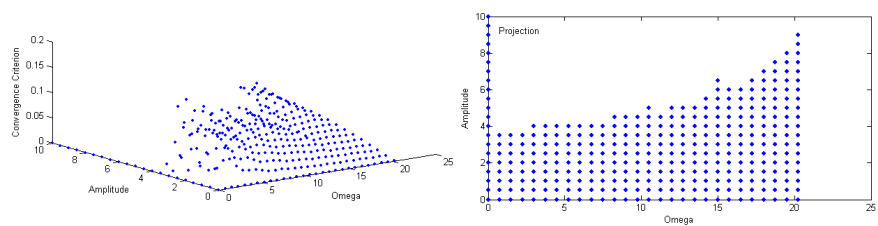

Fig. 9. First selection of the couples parameters

convergence score. Once this first choice is done, we proceed on to the following step which consists in eliminating the sets of parameters which provide mechanical conflicts as it is shown on figure 10. Then we eliminate the sets of parameters which are not in adequation with the links length (figure 10).

\section{B. Optimization according to Criteria}

The research domain is now much more restricted (figure10) and it is possible to launch an exhaustive research on each couple to identify which are those which are best adapted to a desired velocity and to a given optimization criterion (energy, response time, combined). So we can read on figure 11 that if we want to optimize the energy consumption in order to make the system move with for example a velocity of $2.55 \mathrm{~m} . \mathrm{s}^{-1}$, the best choice for the reference curvature is: $C_{i}^{R}=\sin \left(3.75 s_{i}\right)$. We observe that a same couple of parameters can be used for different desired velocities. We have supposed that it was due to the non connected property of the system. The best couples are not selected because they do not belong to the research field. So the selected couples will be the nearest to the definition border, which may explain this curious alignment.

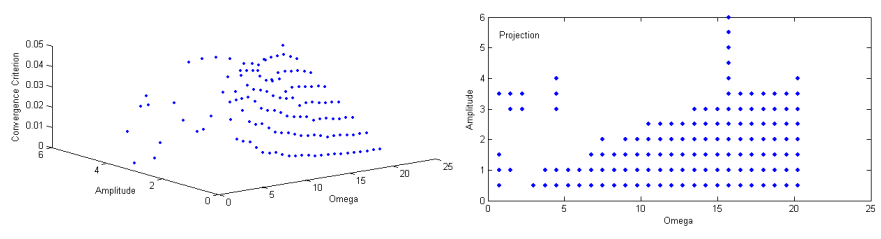

Fig. 10. Second selection of the parameters couples 


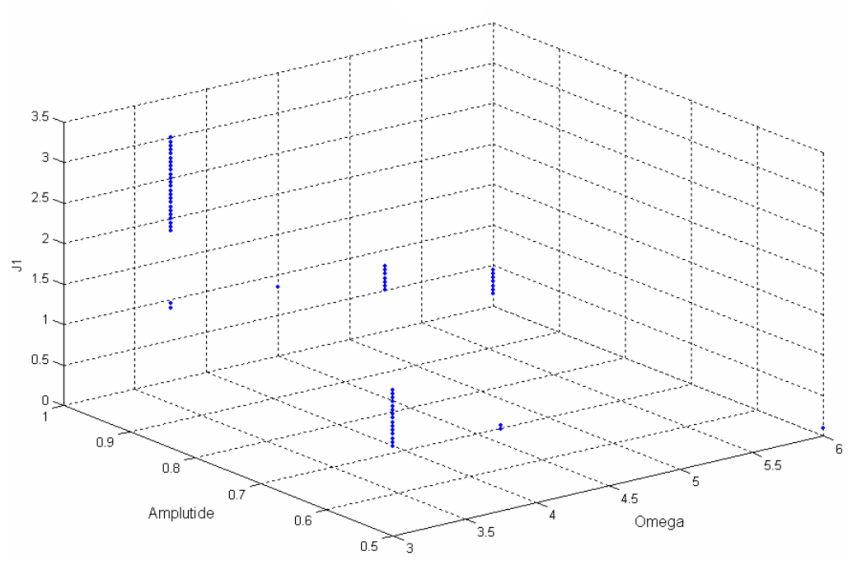

Fig. 11. $J_{1}$ criterion (spent energy)

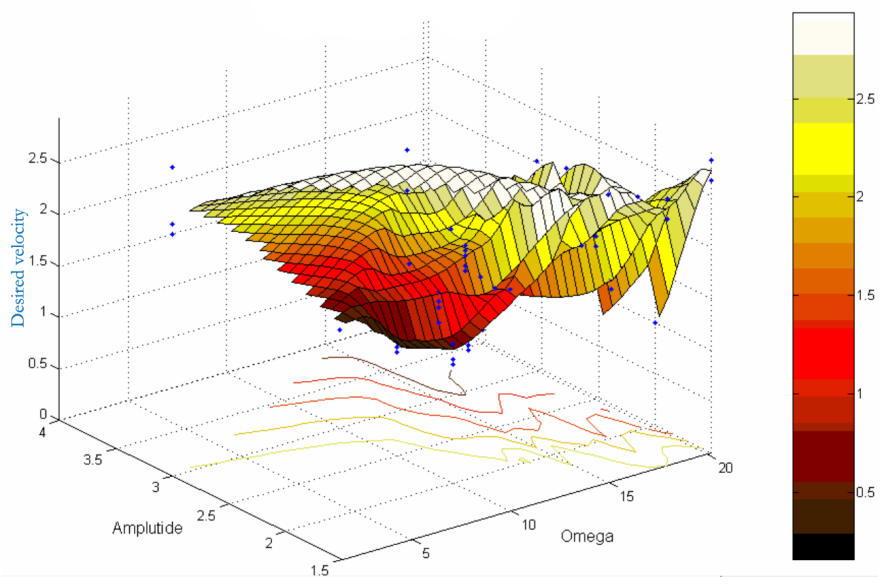

Fig. 12. $J_{2}$ criterion (system time response)

The same approach is used with the $J_{2}$ criterion. We extrapolate a surface from the selected couples. So we can read on figure 12 that to optimize the system response time to make it reach a velocity of $1.45 \mathrm{~m} . \mathrm{s}^{-1}$, the reference curvature must be: $C_{i}^{R}=\sin \left(9 s_{i}\right)$. It's possible to sharpen the appropriate set of parameters because of the injective nature of the graph. Indeed for this criterion several couples correspond to one desired velocity; so it is possible to apply the $J_{1}$ criterion as well, to choose the good one. Although it cannot be accepted as a proof, we are illustrating this method with the example on figure 13. The optimal set of parameters allows the eel to converge towards its desired velocity more quickly $(4.45 \mathrm{~s}$ vs. $7.15 s$ ). The last criterion is $J_{3}$ which is the best compromise between system reaction time and given energy. According to
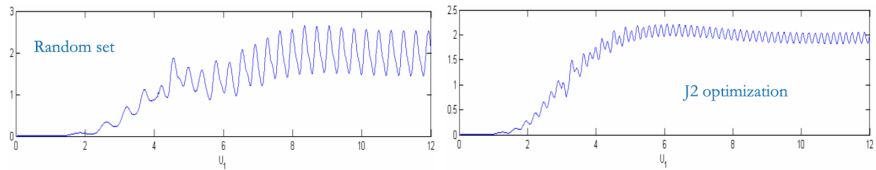

Fig. 13. Different system response time

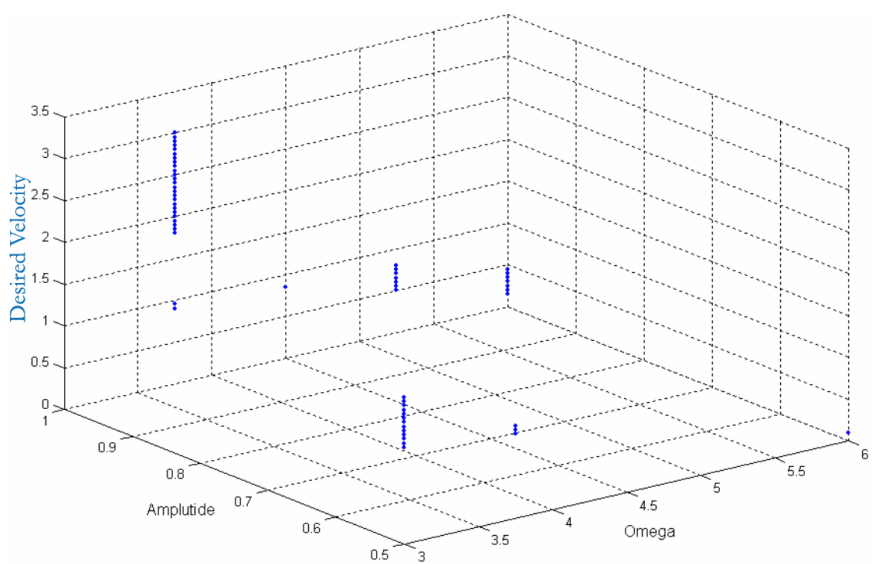

Fig. 14. $\quad J_{3}$ criterion (combined)

figure 14, the best choice for the curvature reference if we wish to optimize the spent energy and the system reaction time in order to reach a velocity of $2.25 \mathrm{~m} . \mathrm{s}^{-1}$, the curvature reference must be: $C_{i}^{R}=\sin \left(3.75 s_{i}\right)$.

\section{Perspectives}

Even if the previous approach improves the choice of the curvature parameters, it does not permit to reach the optimal solution. Indeed this approach is only a local solution which brings progress in a given range of parameters. There may be new directions of research to improve the eel-like robot control.

\section{A. Another Control Equation}

When starting from equation (4) again we can calculate a new $\ddot{\phi}^{R E F}$ by using the Lyapunov method. This leads to a new set of control equations:

$$
\left\{\begin{aligned}
\ddot{\phi}^{R}= & \Xi_{4} \Xi_{2}^{-1}\left(\dot{\nu}_{B}^{d}+K_{\nu}\left(\nu_{B}^{d}-\nu_{B}\right)\right) \\
& +\left(\Xi_{3}-\Xi_{4} \Xi_{2}^{-1} \Xi_{1}\right)\left(f_{1}+B_{1}\right) \\
\ddot{C}_{i}^{R}= & \frac{\ddot{\phi}_{i}^{R}}{d_{i}+d_{i+1}}, \dot{C}_{i}^{R}=\frac{\dot{\phi}_{i}^{R}}{d_{i}+d_{i+1}}, \\
C_{i}^{R}= & \frac{\phi_{i}^{R}}{d_{i}+d_{i+1}} \\
\Gamma= & \Xi_{4}^{-1}\left[\left(\ddot{C}_{i}^{R}+\left(K_{1}+K_{2}\right)\left(\dot{C}_{i}^{R}-\dot{C}_{i}^{E}\right)\right.\right. \\
& \left.+K_{1} K_{2}\left(C_{i}^{R}-C_{i}^{E}\right)\right) \times\left(d_{i}+d_{i+1}\right) \\
& \left.-\Xi_{3}\left(f_{1}+B_{1}\right)\right]-B_{2}
\end{aligned}\right.
$$

where $\dot{\nu}_{B}^{d}, \nu_{B}^{d}, \nu_{B}$ are respectively the head desired acceleration vector, the head desired velocity vector, and the head velocity vector. The thrust is obtained with a periodic signal propagating along the system body. So we have to impose these periodic movements to the head acceleration vector $\left(\dot{\nu}_{B}^{d}\right)$. The system becomes time dependent but on the other hand it seems to be interesting due to the use of all the variables of the system. 


\section{B. Possible Use of Fourier series}

We can also use the Fourier series to generate a thrust for our robot. Indeed we have chosen a sinusoidal curvature (equation III-A.2) arbitrarily but the most general definition of a periodic signal is that of Fourier. It's thus possible to define a new curvature reference as:

$$
C_{i}^{R}=\sum_{n=0}^{k} a_{n} \cos \left(n s_{i} \frac{2 \pi}{T}\right)+\sum_{n=0}^{k} b_{n} \sin \left(n s_{i} \frac{2 \pi}{T}\right)
$$

where $k$ is a positive integer defining the number of coefficients to be used. It's also possible to define the head acceleration vector $\dot{\nu}_{B}^{d}$ like this:

$$
\dot{\nu}_{B}^{d}=\left[\begin{array}{c}
\dot{u}^{d} a_{n} \cos \left(n s_{i} \frac{2 \pi}{T}\right)+\sum_{n=0}^{k} b_{n} \sin \left(n s_{i} \frac{2 \pi}{T}\right) \\
\sum_{n=0}^{k} a_{n}^{\prime} \cos \left(n s_{i} \frac{2 \pi}{T}\right)+\sum_{n=0}^{k} b_{n}^{\prime} \sin \left(n s_{i} \frac{2 \pi}{T}\right)
\end{array}\right]
$$

Now, all the problem consists in determining the coefficients to be used to optimize the shape configuration of the eel robot.

\section{CONCLUSION}

We have described a method to find some good sets of curvature parameters. The previous works of L.Lapierre and B.Jouvencel in [13] have shown that this new type of autonomous gait generation was able to control the local curvature explicitly. This control design is based on Lyapunov methods and guarantees the convergence of the system shape towards the desired curvature profile.

Nevertheless in this work we have shown that some desired curvature profiles were prohibited on pain of seeing the system diverge.The method described here gives insight about the parameters choice to approach system performance. It's possible to create a lookup table in which the control system take good set of parameters. Moreover it can switch in different modes (energy optimization or maximum acceleration for example) and always be able to choose the optimum parameters in the lookup table. Finally this method guarantee a better simulator stability.

This kind of propulsion is expected to be very efficient. From the bio-mimetic inspiration, we could say that a good controller should insure the local fluid flow quality along the body, as fishes do by using a baro-receptors line distributed all along their body. From now on, the existing solutions impose a predefined gait that guides the system shape, and induces propulsion. Then the system efficiency is directly dependent on this gait choice. Nevertheless, we are working now on a gait adaptation strategy in order to combine a nominal shape respect by taking local forces control into consideration. Consequently this work could be used to study the propulsion system efficiency explicitly and to compare it to existing solutions based on propellers.

\section{REFERENCES}

[1] Muller, Ulrike K., Stamhuis, Eize J., Videler[dagger], John J. Riding the Waves: The Role of the Body Wave in Undulatory Fish Swimming., Nov 2002.

[2] Index of the site ROBEA: Robot Anguille, Informations avaible on: http://www.irccyn.ec-nantes.fr/hebergement/ROBEA, Sept 2004.

[3] Ayers, J., Wilbur, C., Olcott, Lamprey Robots. In: Proceedings of the International Symposium on Aqua Biomechanisms., T. Wu and $\mathrm{N}$, Kato, [eds]. Tokai University., 2000.

[4] Professor Jim Ostrowski (GRASP Lab), and Kenneth McIsaac (GRASP Lab), Designng an Underwater Eel-like Robot ans Developing Anguilliform Locomotion Control, Tamara Knutsen, Harvard University, Sunfest Projects, Summer 2000.

[5] K. McIsaac and J. Ostrowski, A Geometric Approach to Anguilliform Locomotion: Modeling of an Underwater Eel Robot, in Proc. Of the IEEE Conference on Robotics and Automation (ICRA 99), pp. 28433848, Detroit Michigan, USA, May 10-14, 1999.

[6] H. Vela, K. Morgansen and J. Burdick, Underwater locomotion from oscillatory shape deformations, in Proc. of the 41st IEEE Conference on Decision and Control (CDC), Las Vegas, Nevada USA, December 2002, pp 2074-2080.

[7] K. McIsaac and J. Ostrowski, Motion Planning for Anguiliform Locomotion, in IEEE Transaction on Robotics and Automation, August 2003, 19(4), pp 637-652.

[8] J. Ostrowski and K. McIsaac, Open-loop Verification of Motion Planning for an Underwater Eel-like Robot, in Proc. of the 7th International Symposium on Experimental Robotics (ISER), Honolulu, Hawaii, December $10-13,2000$

[9] W. Saintval, optimizing robotic aquatic locomotion, in Proc of the Thirty-fourth Annual Meeting of the Florida Section of the Mathematical Association of America, David Kerr and Bill Rush eds, April 2002, Florida, USA.

[10] D. Sfakiotakis and M. Tsakiris, A simulation Environement for Undulatory Locomotion, in Proc. of the International Conference on Applied Simulation and Modelling, June 28-30, 2004, Rhodes Island, Greece.

[11] O. Ekeberg, A Combined Neuronal and Mechanical Model of Fish Swimming, Biological cybernetics, Vol. 69, pp 363-374, 1993.

[12] T. Fossen, Guidance and Control of Ocean Vehicles, John Willey \& Sons Inc, New York, 1994.

[13] L. Lapierre and B. Jouvencel, Path Following Control for an Eel-like Robot, Ocean2005, Brest, June 2005. 